\title{
Evaluación Morfométrica de la Relación Embrio-Uterina de las Etapas Pre y Post Implantacional en Conejo (Oryctolagus cuniculus)
}

\author{
Morphometric Evaluation of the Embryo-Uterine Relationship of the \\ Pre and Post Implantational Stages in Rabbit (Oryctolagus cuniculus) \\ "Carolina Schencke; ${ }^{* *}$ Mariana Rojas \& ${ }^{* * *}$ Mariano del Sol
}

SCHENCKE, C; ROJAS, M \& DEL SOL, M. Evaluación morfométrica de la relación embrio-uterina de las etapas pre y post implantacional en conejo (Oryctolagus cuniculus). Int. J. Morphol., 26(4):995-1004, 2008.

RESUMEN: El conejo ha demostrado ser un excelente modelo de estudio de implantación. Como ovulador obligado, el tiempo de preñez se puede establecer en forma precisa. La observación morfológica de cortes de úteros de 7, 8, 9 y 10 días pos coito, permitió en este estudio reconstruir una secuencia de los eventos morfométricos que ocurren durante la implantación, en el conejo. Se utilizaron 20 conejas neozelandesas blancas adultas (Oryctolagus cuniculus), nulíparas, no gestantes. Para la cruza, se utilizaron 5 machos de probada fertilidad. Los conejos fueron obtenidos del Bioterio de la Facultad de Medicina de la Universidad de La Frontera, Temuco, Chile. Determinamos como día 0, el momento del coito, sacrificando las hembras los días 7, 8, 9 y 10 de cada cruza. Una vez sacrificados, se disecó macro y mesoscópicamente la región pélvica, seleccionándose vesículas uterinas para sus estudios histológico e inmunocitoquímico. Se realizaron estudios morfométricos y de cinética celular con las técnicas de Tunel y PCNA. Se efectuó estadística descriptiva en base a promedio y desviación estándar $(\mathrm{p}<0.001)$, utilizando el programa estadístico Stata 9.0. Las mediciones morfométricas obtenidas fueron de diámetro y volumen de la vesícula uterina, altura de la pared uterina y lumen glandular de regiones mesometrial y antimesometrial, para los días 7 al 10 post coito. Los análisis inmunocitoquímicos permitieron determinar: índice apoptótico de los núcleos celulares y el índice celular mitótico. Para el día 8 post coito se observaron los cambios morfométricos más significativos a nivel del lumen vesicular, altura de pared uterina de la región mesometrial, y lumen glandular. El mismo día se observaron cambios importantes en los índices celular mitótico y apoptótico. El conejo podría ser una especie usada para predecir el normal desarrollo embrionario, tras la comprensión morfológica y morfométrica de la implantación. Presenta una forma poco invasiva de implantación y una capacidad de formación placentaria temprana. Este estudio permite una mejor comprensión del mecanismo implantacional y ayudada a proveer información de actualización en el desarrollo normal del embrión de conejo.

PALABRAS CLAVE: Morfmetría; Relación embriouterina; Implantación; Conejo.

\section{INTRODUCCIÓN}

En la actualidad, es posible controlar y mejorar la mayoría de las fases del proceso reproductor, exceptuando la capacidad del embrión para implantarse en el endometrio materno. Este proceso podría definirse como un estado de transición en el progreso de la preñez, durante el cual el blastocisto se fija al endometrio materno y comienza una relación embriouterina. El conejo ha demostrado ser un excelente modelo de estudio de la implantación. Al actuar como ovulador obligado, logrando establecerse de manera precisa el tiempo de preñez. Recientemente, se ha progresado en el estudio de las interacciones celulares y mecanismos fisiológicos envueltos en la iniciación de la implantación, tanto en mamíferos como el conejo. Pero, a su vez, es importante realizar una vista general sobre los contextos morfológico y morfométrico de la implantación, y lograr discernir varios aspectos de la relación embrio-uterina.

Estudiamos los cambios estructurales que implican la maduración funcional del endometrio durante la gestación temprana. Esto involucra el análisis de procesos de proliferación celular y apoptosis de poblaciones celulares del epitelio uterino secretor glandular y de revestimiento.

\footnotetext{
* Escuela de Ciencias de la Salud, Universidad Autónoma de Chile, Temuco, Chile.

** Facultad de Medicina, Universidad de Chile, Santiago, Chile.

**** Facultad de Medicina, Universidad de La Frontera, Temuco, Chile.
} 
Con propósitos comparativos, se han incluido los resultados concernientes a la morfología implantacional en otras especies de mamíferos, tales como ratas, ratones y otros roedores. Pitt \& Carney, (1999), realizaron estudios morfológicos de la etapa postimplantacional en conejos neozelandeses blancos, estableciendo así el arquetipo normal de desarrollo entre los días 9 al 13 post coito (p.c.). Describieron que, en general, muchos de los patrones de desarrollo son similares a los de roedores. Una de las primeras notables diferencias en la implantación, es que en el día 13, el embrión de conejo no está completamente envuelto por el saco vitelino, como en rata y ratón. Así, la placenta estaría exhibiendo un crecimiento extensivo si se compara con los estadios tempranos en de especies roedores. Señalaron que el conejo ya ha formado dos largos discos placentarios, aún cuando no está presente la placenta madura. La segunda diferencia que observaron es que la orientación del embrión de conejo es distinta de la presentada por roedores. El día 9 p.c., el embrión de conejo yace como un disco alargado con su lado dorsal hacia la cama placentaria.

Parr et al., (1987) reportaron cambios autolíticos antes de ser fagocitados por el trofoblasto. La degeneración del epitelio uterino que rodea la cámara de implantación, en rata y ratón comienza en la región antimesometrial y se continúa con la región mesometrial, la que aún no está en contacto directo con el embrión. La apoptosis ha sido considerada como suicidio fisiológico progamado. Según Ferro \& Bevilacqua, (1994), en el roedor Calomys callosus, la degeneración epitelial parece ser un proceso apoptotico, estableciendo rápidamente áreas rodeadas por el trofoblasto. Esto podría ser resultado de una interferencia activada por el trofoblasto para facilitar el proceso de invasión.

Goodger \& Rogers, (1993), realizaron estudios de la proliferación de las células endometriales en la implantación de ratas. Usando inmunohistoquímica con antígeno nuclear de proliferación celular (PCNA) cuantificaron la proliferación celular. El índice de proliferación celular endometrial mostró un $1 \%$ en los primeros 2 días de preñez y un $13 \%$ en el día 3 . El aumento continuó a un $28 \%$ en el día 5 de preñez. Luego de la implantación embrionaria, el índice de proliferación de las células endoteliales llegó al $71 \%$ en el día 7 de gestación, pero sólo en el sitio de implantación del embrión, disminuyendo significativamente en las regiones adyacentes a este sitio. Por lo tanto, según estos autores, el índice de proliferación endometrial tiene valores que aumentan en un $22 \%$ en la cámara de implantación, durante la etapa preimplantacional.

Ohta et al., (1993), realizaron un estudio sobre el desarrollo de las glándulas endometriales en útero de rata entre los días 8 al 16 de preñez. En la decidua basal, las células de las glándulas endometriales incrementaron su número lentamente durante los días 8 al 12 de preñez, para luego decrecer. El incremento celular continuaba desde el día 9 al 16 de preñez, formando las glándulas endometriales. Los autores concluyeron que las células negativas para la técnica del ácido peryódico de Schiff (PAS) y que mostraban una mitosis activa, eran presumiblemente, precursores de células de glándulas endometriales.

Conti et al., (1984), señalaron que los estrógenos modificaban la cinética de las células epiteliales glandulares del endometrio, las que normalmente migran hacia el lumen. El efecto estrogénico en hembras no gestantes, se manifiesta en una menor pérdida de células luminales y mayor proliferación epitelial. Concluyeron que las señales del blastocisto estarían induciendo la cascada apoptótica, y así también, genes estarían regulando este evento local. Señalaron además que estas regulaciones son aún desconocidas en conejo durante el período implantacional.

Según Galán et al., (2000), Aplin \& Kimber (2004), la apoptosis o muerte celular programada es un proceso fisiológico importante, especialmente en los sitios que requieren una continua renovación celular como las mucosas intestinal y uterina. Estos autores indican que el embrión humano induce la apoptosis de las células epiteliales endometriales localizadas en el lugar de adhesión, facilitando la entrada del embrión en el estroma endometrial. El contacto con la superficie epitelial endometrial, es seguido por apoptosis, permitiendo la etapa de penetración embrionaria.

Anzaldúa et al., (2001), analizaron la dinámica de los índices de proliferación celular y apotosis en el período preimplantacional del endometrio de conejas neozelandeses, observando incrementos del índice mitótico acompañados de una marcada disminución del índice apoptótico entre los días 2 y 5 de la gestación. Describieron que durante la gestación temprana de diversos mamíferos es común la ocurrencia de picos en el índice mitótico. Estos días de mayor incremento mitótico se dan de manera temprana en el útero, entre los días 2 y 3 de gestación.

Joswig et al., (2003) y Akcali et al., (2003), estudiaron la apoptosis en el epitelio uterino de ratón durante los días 4 al 7 p.c., mediante la técnica de TUNEL (terminal deoxynucleotide transferase mediated dUTP-biotin nick end Labeling). Indicaron que durante las etapas iniciales de la implantación en ratón, la cámara implantacional se encuentra con apoptosis, en respuesta a la interacción con el blastocisto. La degeneración del epitelio uterino que rodea el sitio de implantación comienza en la región antimesometrial, seguida luego por la región mesometrial. Las primeras señales de dobles bandas de DNA fueron de- 
tectadas en el día 5 p.c. en el epitelio uterino de la cámara implantacional. En el compartimiento estromal observaron apoptosis en el día 6 p.c. en la zona decidual primaria.

Schencke et al., (2004), describen morfológicamente la implantación embrionaria en conejo (Oryctolagus cuniculus). El embrión de conejo se posiciona en la región antimesometrial, el fenómeno de adhesión se observa mediante botones trofoblásticos en la región antimesometrial, y la implantación del embrión (ICM) se observa en la región mesometrial.

Según Wang \& Dey (2006), en su estudio sobre el mecanismo de implantación embrionaria en ratón, las células estromales que rodean al blastocisto activan el proceso de decidualización en el polo antimesometrial, orientando la cámara implantacional en sentido antimesometrialmesometrial. Según Hondo et al., (2007), en el proceso de implantación de las distintas especies de mamíferos, las diferencias morfológicas y funcionales se observan a nivel del epitelio luminal. El embrión de ratón se implanta en la región donde se ubican las glándulas uterinas, localizadas en la región antimesometrial. Este estudio sugiere que las glándulas uterinas guían al embrión al lugar más apropiado, el que a su vez puede estar programado genéticamente.

Los estudios de Ghaffari et. al. (2007) se enfocan en células epitelilaes endometriales, las que juegan un rol crucial en la adhesión embrionaria. Cuando inducen la ovulación en la fase lútea, resulta en un incremento en la actividades secretoria y metabólica de las células epiteliales endometriales. Según estudios realizados por Lindeberg, (2008), en ferret, el embrión se implantaría en el día 12 a 13 post coito. La implantación en este mamífero es central, invadiendo el epitelio endometrial, sin destruir el endotelio de los capilares maternales, como sucede en rata, ratón y otros roedores.

\section{MATERIAL Y MÉTODO}

Con el objetivo de analizar la interrelación embrionaria pre y post implantacional en conejo, se utilizaron 20 conejas Neozelandesas (Oryctolagus cuniculus), adultas, nulíparas, no gestantes, clínicamente sanas, $(3,5$ - 4,5kg de peso), criadas y mantenidas en cautiverio, alimentadas con pellets y zanahorias ad libitum. Para la cruza, se utilizaron 5 conejos neozelandeses (Oryctolagus cuniculus), machos, de probada fertilidad. Los ejemplares en edad reproductiva se encontraban clínicamente sanos, fueron criados y mantenidos solitarios en cautiverio. Los conejos fueron obtenidos del Bioterio de la Facultad de Medicina de la Universidad de La Frontera, Temuco, Chile.
Cronología de las cruzas. Se determinó como día 0, el momento del coito, sacrificando las hembras los días 7, 8, 9 y 10 de cada cruza. Para esto, las conejas fueron divididas en cuatro grupos de cinco ejemplares cada uno. El grupo 1 se sacrificó a los 7 días p.c., el grupo 2 a los 8 días p.c., el grupo 3 a los 9 días p.c., y el grupo 4 a los 10 días p.c.

Obtención de las muestras. Una vez sacrificadas las conejas, se disecó macro y mesoscópicamente la región pélvica, extrayéndose los úteros (Fig. 1). El útero de cada coneja gestante fue removido y depositado en buffer fosfato salino (PBS). Se cuantificaron el número de implantes en cada útero, y la normalidad de cada embrión. Las muestras fueron mantenidas húmedas con suero fisiológico estéril mientras se realizaba la disección. Los úteros derecho e izquierdo fueron aislados, depositándose cada uno por separado en PBS (Fig. 2). Posteriormente, se seleccionaron vesículas de cada útero. Las vesículas del útero derecho se colocaron en formalina al $10 \%$ y se usaron para realizar los estudios histológicos, histoquímicas y morfométricos. Las vesículas uterinas izquierdas se colocaron en methacarn y se usaron para el estudio inmunohistoquímico. Las disecciones se realizaron con la ayuda de una lupa ransor (10X). Las vesículas uterinas de conejo se fotografiaron con una lupa Olympus SZ-PT, con cámara OLYMPUS C-35DA-2.

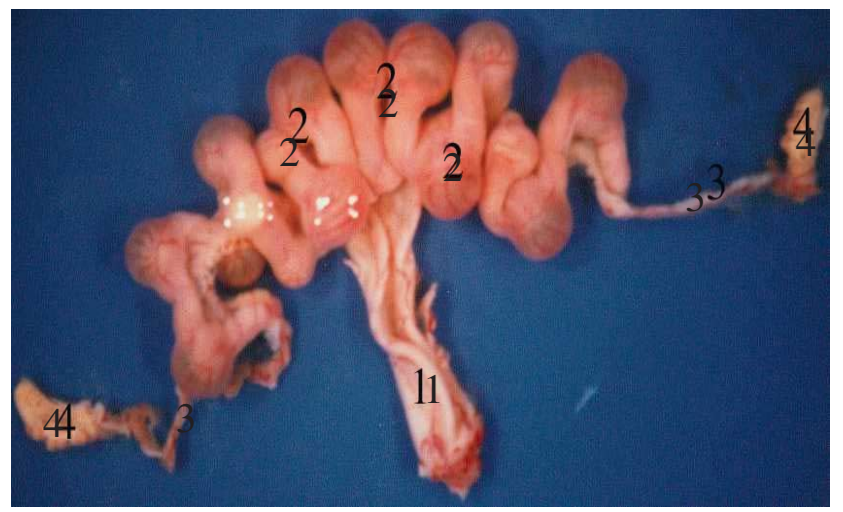

Fig. 1. Se observa el útero doble de coneja Oryctolagus cuniculus, con la presencia de vesículas uterinas que contienen embriones del día 9 pos coito. 1. Vagina; 2, Útero (Vesículas uterinas); 3 Tuba uterina; 4. Ovarios.

Análisis morfométrico. Para el análisis morfométrico se efectuó estadística descriptiva en base a promedio (X) y desviación estándar (DS), n = número de casos, calculados para cada una de las variables analizadas. Se realizó un análisis morfométrico del diámetro, lumen y altura de las vesículas embrionarias, usándose lupa con regla de calibración. Además, se midieron las regiones mesometrial, antimesometrial, y la base y lumen glandular de cada región. Con este fin, se analizaron 10 placas para cada conejo estudiado, en los días 7 al 10 p.c., con placa de calibración. 


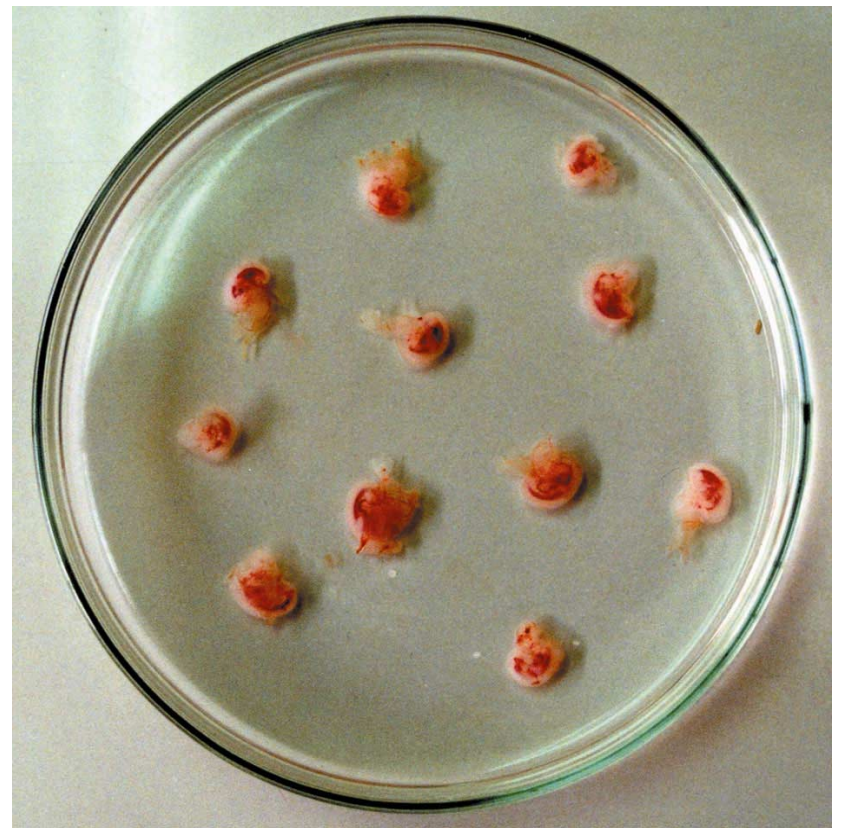

Fig. 2. Vesículas uterinas de coneja del día 9 pos coito, aisladas y colocadas en placa Petri en PBS.

Para comprobar si existía diferencia estadísticamente significativa entre las variables medidas, para los días 7 al 10 p.c., se utilizó un análisis de tendencia entre grupos ordenados, aplicando el Test de Cuzik no paramétrico, el cual es una extensión del Test de Wilcoxon de sumas de rangos. Todos estos análisis se realizaron utilizando el programa estadístico STATA 9.0.

Análisis inmunocitoquímico. Se efectuó con las muestras fijadas en methacarn, las cuales fueron tratadas con diferentes anticuerpos para la identificación de Ag, tanto en células endometriales como en células del embrión. Se sometieron a cada una de estas técnicas, 10 placas por conejo para cada día p.c. En la técnica inmunocitoquímica de PCNA para identificar proliferación celular, se utilizó el anticuerpo primario: anti PCNA. Para la detección de la fragmentación de DNA in situ, se utilizó la técnica TUNEL (Terminal transfferasa dUTP nickend Labeling), Roche (Basel Switzerland).

Con la técnica de PCNA, la inmunoreacción marcó de color café los núcleos que se encontraban en las etapas de división celular. El índice de proliferación celular (ICM) se cuantificó con la técnica de PCNA en los epitelios luminal y glandular de ambas regiones endometriales. Se calculó el porcentaje de células con inmunotinción positiva para PCNA, en 10 campos en 400X. Los resultados de la cuantificación se expresaron como porcentajes de células en mitosis del total de células. Se obtuvieron promedios y desviación estándar.
Con la técnica de TUNEL la inmunorreacción marcó de color café los núcleos apoptóticos. Con el microscopio de luz, se contaron los núcleos de los epitelios luminal y glandular con inmunoreacción positiva para TUNEL. Se calculó el porcentaje de células con inmunotinción positiva para la técnica de TUNEL en 10 campos en 400X. Los resultados de la cuantificación se expresaron como porcentaje de células en apoptosis del total de células.

Se obtuvieron promedios y desviación estándar, índice celular mitótico (ICM) e índice apoptotico (IA) en células epiteliales de revestimiento y conjuntivas en ambas regiones mesometriales. Se consideró un índice de significancia de 0.01 (probabilidad del 99\%).

\section{RESULTADOS}

Tanto para la localización del embrión y del trofoblasto utilizamos los términos de regiones mesometrial y antimesometrial en las vesículas uterinas, con fines didácticos de orientación espacial, entendiéndose que estas regiones comprenden el perimetrio, el miometrio y el endometrio. Por tanto, la región mesometrial comprendería el área de penetración del pedículo vascular a la vesícula uterina, considerándose el resto de la vesícula como la región antimesometrial.

Análisis Morfométrico. Fueron calculados promedio y desviación estándar, para las mediciones obtenidas del diámetro y lumen de la vesícula uterina, altura de la pared uterina en la región mesometrial (PM) y de la pared uterina en la región antimesometrial (PAM), además del lumen glandular en las regiones mesometrial y antimesometrial, para los días 7 al 10 p.c., como se muestra en las Tablas I a III.

El diámetro de la vesícula embrionaria aumenta aproximadamente, $2 \mathrm{~mm}$ cada día p.c. El lumen vesicular es muy pequeño en el día 7 p.c. experimentando un aumento significativo el día 8 p.c. La altura de la pared uterina en la región antimesometrial disminuye considerablemente entre el día 7 al día 8 p.c. La altura de la pared uterina en la región mesometrial aumenta entre los días 7 al 9 p.c., manteniendo su altura entre los días 9 y 10 p.c.

El promedio del lumen glandular en la región mesometrial tiene un aumento considerable entre los días 7 al 10 p.c. Las glándulas son alargadas y de lumen regular en el día 7 p.c. El lumen glandular de la región antimesometrial aumenta considerablemente en el día 8 p.c. en la región mesometrial, en cambio, aumenta en el día 9 p.c. 
Tabla I. Crecimiento de las vesículas uterinas de coneja Oryctolagus cuniculus (7, 8, 9 y 10 días p.c.).

\begin{tabular}{ccccc}
\hline Días p. c. & $\begin{array}{c}\text { X Diámetro vesícula } \\
\text { uterina }(\mathbf{m m})\end{array}$ & DS & $\begin{array}{c}\text { X Lumen vesícula } \\
\text { uterina }(\mathbf{m m})\end{array}$ & DS \\
\hline 7 & 6,20 & 0,23 & 1,95 & 0,12 \\
8 & 9,02 & 0,36 & 6,53 & 0,22 \\
9 & 12,15 & 0,29 & 7,08 & 0,20 \\
10 & 14,29 & 0,37 & 8,95 & 0,11 \\
\hline
\end{tabular}

Tabla II. Altura de las paredes uterinas de las regiones mesometrial y antimesometrial de coneja Oryctolagus cuniculus a los 7, 8, 9 y 10 días p.c.

\begin{tabular}{ccccc}
\hline Días p. c. & $\begin{array}{c}\text { X Altura PAM } \\
(\mathbf{m m})\end{array}$ & DS & $\begin{array}{c}\text { X Altura PM } \\
(\mathbf{m m})\end{array}$ & DS \\
\hline 7 & 1,45 & 0,12 & 2,96 & 0,08 \\
8 & 0,44 & 0,06 & 3,16 & 0,06 \\
9 & 0,31 & 0,09 & 5,09 & 0,05 \\
10 & 0,28 & 0,06 & 5,12 & 0,09 \\
\hline
\end{tabular}

$\mathrm{PAM}=$ Pared antimesometrial $\mathrm{PM}=$ Pared mesometrial

Tabla III. Lumen glandular en las regiones mesometrial y antimesometrial del endometrio de la coneja Oryctolagus cuniculus, durante los días 7, 8, 9 y 10 p.c.

\begin{tabular}{ccccc}
\hline Días p. c. & $\begin{array}{c}\text { X Lumen } \\
\text { glandular PAM } \\
(\boldsymbol{\mu m})\end{array}$ & DS & $\begin{array}{c}\text { X Lumen } \\
\text { glandular PM } \\
(\boldsymbol{\mu m})\end{array}$ & DS \\
\hline 7 & 50,03 & 0,12 & 30,15 & 0,16 \\
8 & 139,73 & 0,89 & 35,04 & 0,19 \\
9 & 170,33 & 0,24 & 70,49 & 0,30 \\
10 & 210,28 & 0,16 & 75,24 & 0,27 \\
\hline
\end{tabular}

PAM= Pared antimesometrial $\mathrm{PM}=$ Pared mesometrial

Tabla IV. Índice celular mitótico en células (ICM) del epitelio glandular y en células conjuntivas endometriales durante los días 7, 8, 9 y 10 p.c., analizadas en 50 placas histológicas de coneja Oryctolagus cuniculus.

\begin{tabular}{ccccc}
\hline Días p. c. & $\begin{array}{c}\text { X ICM en células del } \\
\text { epitelio glandular }\end{array}$ & SD & $\begin{array}{c}\text { X ICM en células } \\
\text { conjuntivas }\end{array}$ & SD \\
\hline 7 & 91,09 & 0,21 & 69,41 & 0,44 \\
8 & 50,63 & 0,34 & 30,19 & 0,17 \\
9 & 39,32 & 0,25 & 20,71 & 2,45 \\
10 & 41,43 & 0,39 & 18,29 & 0,19 \\
\hline
\end{tabular}

Tabla V. Índice celular apoptótico (IA) analizado en células del epitelio glandular y en células conjuntivas endometriales en coneja Oryctolagus cuniculus durante los días 7, 8, 9 y 10 p.c. analizadas en 50 placas histológicas.

\begin{tabular}{rcccc}
\hline Días p. c. & $\begin{array}{c}\text { X IA en células del } \\
\text { epitelio glandular } \\
40,18\end{array}$ & SD & $\begin{array}{c}\text { X IA en células } \\
\text { conjuntivas }\end{array}$ & SD \\
7 & 70,43 & 0,16 & 40,10 & 0,19 \\
8 & 85,38 & 0,15 & 65,19 & 0,20 \\
9 & 91,13 & 0,14 & 75,07 & 0,20 \\
10 & 0,24 & 90,89 & 0,60 \\
\hline
\end{tabular}

Mitosis y apoptosis celular. El análisis inmunocitoquímico con la técnica de TUNEL, permite identificar las células apoptóticas, incluso las que se encuentran en estadíos muy iniciales de marginación cromatínica. Las células con reacción positiva para TUNEL presentaron color café oscuro, que representa las roturas de ADN cromosómico. Esta técnica permitió determinar el IA (indice apoptótico) de los núcleos celulares, tanto de las células del epitelio glandular como en células conjuntivas (estromales) del endometrio de coneja Oryctolagus cuniculus. La técnica inmunocitoquímica de PCNA permitió determinar el ICM (índice celular mitótico) de los núcleos celulares en célulaas conjuntivas endometriales y del epitelio glandular. Los núcleos que se encontraban en división celular se observaron de color café. Se calcularon el promedio y desviación estándar del ICM durante los días 7 al 10 p.c., como se muestra en las Tablas IV y V.

Se observa una disminución del índice celular mitótico para los días 7 al 10 p.c., tanto en epitelio glandular, como en células conjuntivas del epitelio endometrial a nivel luminal. Esta disminución es notoria en el día 8 p.c. para ambos grupos celulares endometriales, (Tabla IV).

El índice celular apoptótico aumenta durante los días 7 al 10 p.c. Este aumento es considerable en el día 8 p.c. para ambos grupos celulares endometriales estudiados. Cuando existe apoptosis a nivel del epitelio glandular también existe en las células endometriales a nivel luminal en porcentajes similares (Tabla V). A medida que avanzan los días de gestación, disminuyen las mitosis, y aumentan las apoptosis, durante el periodo estudiado. 

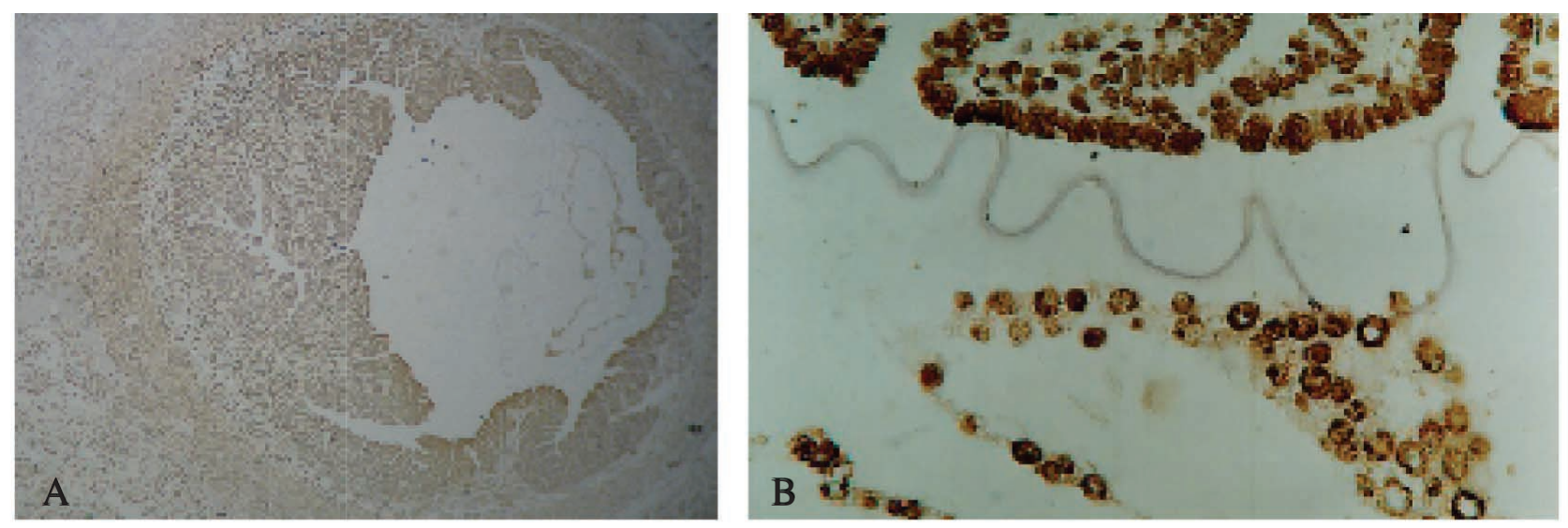

Fig. 3. Día 7 p.c. mostrando inmunotinción para la técnica de TUNEL. A. Útero de coneja Oryctolagus cuniculus, con vesícula embrionaria libre en el lumen en el día 7 p.c. Lupa. B. El embrión presenta inmunopositividad para la técnica de TUNEL, y se encuentra rodeado por la capa mucoide. TUNEL. 400X.

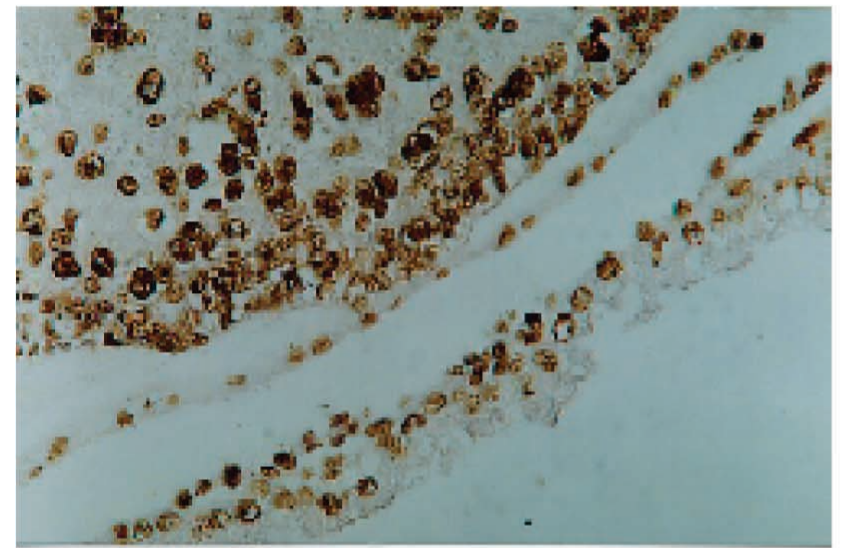

Fig. 4. Día 8 p.c. mostrando inmunotinción para la técnica de TUNEL. 1. Trofoblasto implantándose en el endometrio de coneja Oryctolagus cuniculus, con inmunotinción positiva para la técnica de TUNEL. 400X.

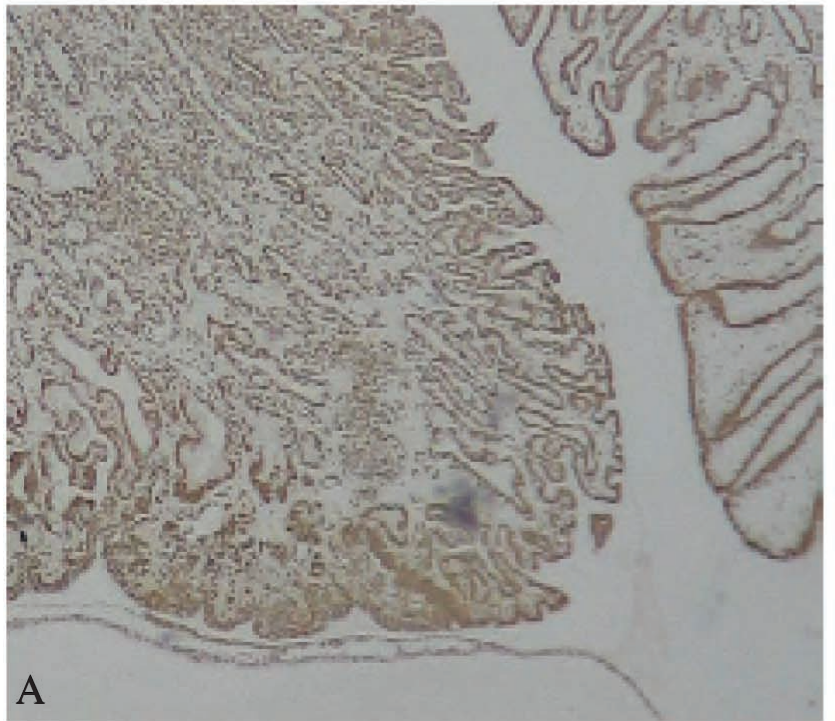

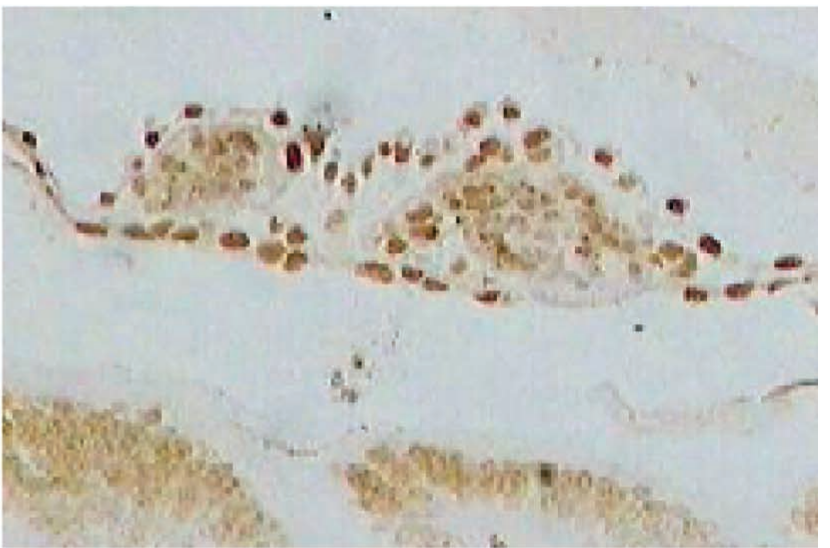

Fig. 5. Día 8 p.c. mostrando inmunotinción para la técnica de TUNEL. 1. Botones trofoblásticos de coneja Oryctolagus cuniculus cercanos al epitelio luminal endometrial. TUNEL. 400X.

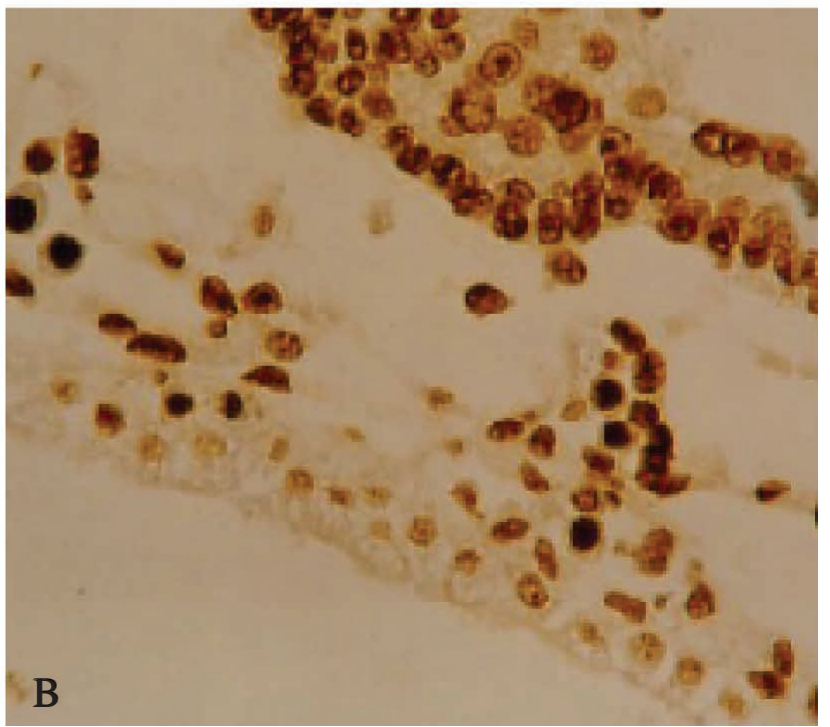

Fig. 6. Día 9 p.c. mostrando inmunotinción positiva para la técnica de TUNEL. A. Pliegue de la mucosa endometrial de coneja Oryctolagus cuniculus. B. Relación embrio-uterina. 1. Trofoblasto. 2. Endometrio. TUNEL. 400X. 

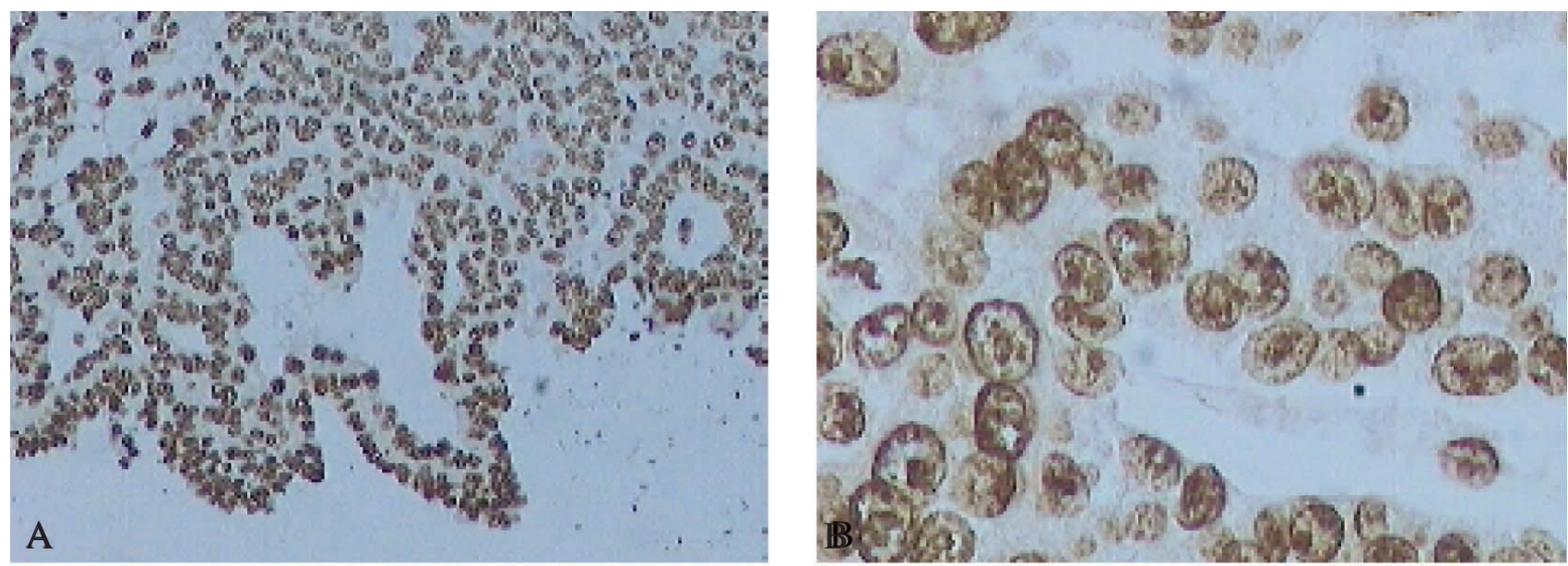

Fig. 7. Día 10 p.c. mostrando inmunotinción positiva para la técnica de TUNEL. A. Células del estroma endometrial de conejo Oryctolagus cuniculus con inmunotinción positiva para la técnica de TUNEL, en el día 10 p.c. 100X. B. Células del estroma endometrial con inmunotinción positiva para la técnica de TUNEL. 400X.

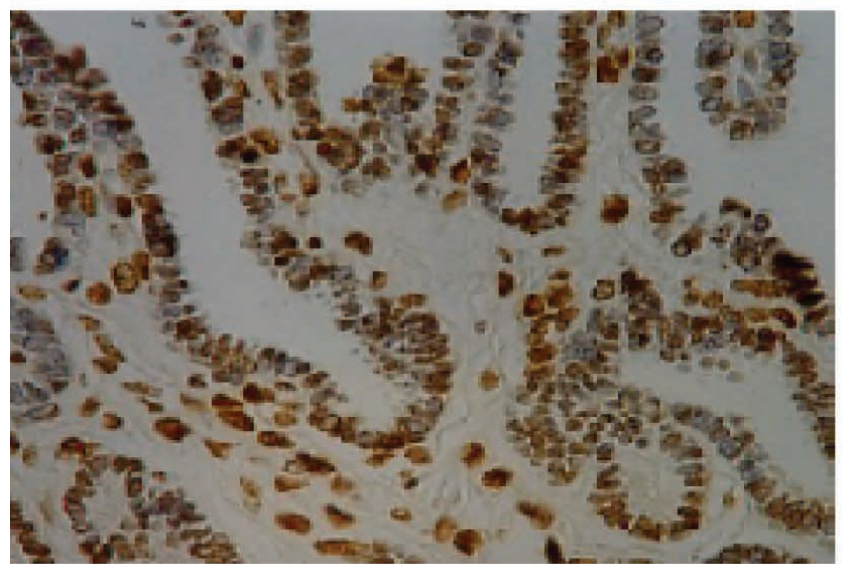

Fig. 8. Día 7 p.c. mostrando inmunotinción para la técnica de PCNA de la mucosa endometrial de coneja Oryctolagus cuniculus. 1. Células conjuntivas del estroma endometrial con inmunotinción positiva. 2. Células del epitelio glandular con inmunotinción positiva para la técnica de PCNA. 400X.

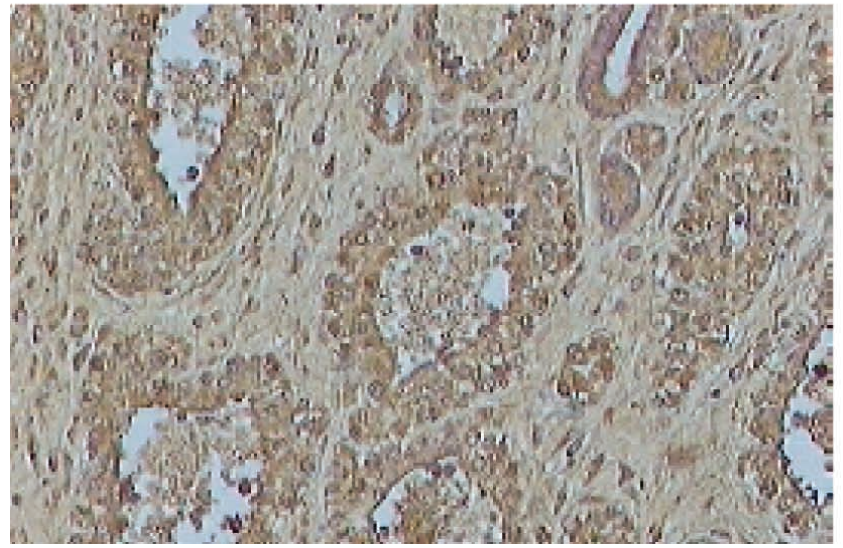

Fig. 10. Día 9 p.c. mostrando estroma endometrial de coneja Oryctolagus cuniculus con inmunotinción positiva para la técnica de PCNA. 400X.

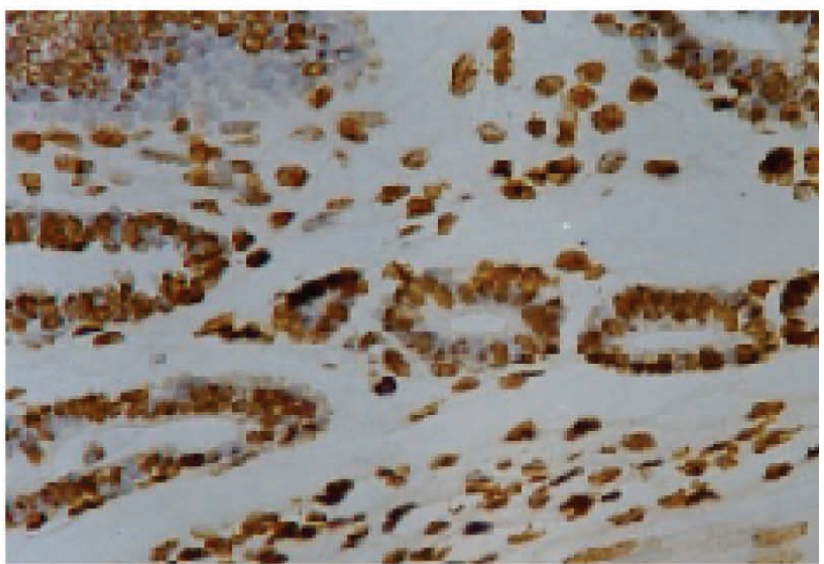

Fig. 9. Día 8 p.c. mostrando inmunotinción para la técnica de PCNA de la mucosa endometrial de coneja Oryctolagus cuniculus. 1. Células conjuntivas del estroma endometrial presentando inmunotinción positiva para la técnica de PCNA, 2. Células del epitelio glandular presentando inmunotinción positiva para PCNA. 400X.

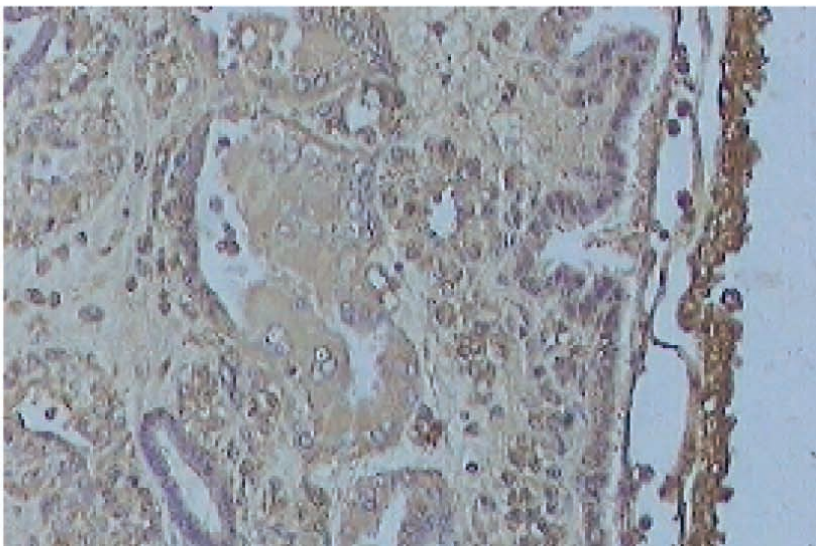

Fig. 11. Día 10 p. c. Se observan algunas células conjuntivas del estroma endometrial con inmunotinción positiva para la técnica de PCNA. 2. Glándula presentando inmunotinción debilmente positiva para PCNA. 100X. 
En las placas evaluadas se observó una tinción específica para los núcleos albos (Figs. 3 a 11). En el día 7 y 8 p.c. se observa la invasión del trofoblasto y la muerte programada de células epiteliales glandulares y células conjuntivas estromales cercanas al lumen (Figs. 3 a 7). A su vez, la proliferación celular endometrial disminuye a nivel de los epitelios luminal y glandular, (Figs. 8 a 11). En el día 9 p.c. el estroma se torna edematoso. Rodeando las glándulas se observa una gran proliferación celular (Fig. 10). En el día 10 p.c. se observa una escasa proliferación celular, tanto a nivel de las células del epitelio glandular como en las células estromales de la región luminal (Fig. 11).

\section{DISCUSIÓN}

Evaluación morfométrica de la relación embrio-uterina. Aunque el conejo es un modelo popular de estudio embriológico, existen muy pocos análisis morfológicos acerca del desarrollo pre y post implantacional normal, especialmente durante los días 7 al 10 p.c. En el proceso de la implantación embrionaria, el endometrio de coneja Oryctolagus cuniculus pasa por cambios morfológicos secuenciales, los que son más significativos cuando la implantación está por ocurrir. Si observamos ambas regiones endometriales, es posible notar que existen cambios morfométricos que delatan el crecimiento del embrión en la cámara implantacional. La presencia del embrión influye sobre la región mesometrial, en donde se observan abundantes pliegues de la mucosa uterina y tejido glandular, lo que se presenta en menor grado en la región antimesometrial. Es así como el lumen glandular observado en la región mesometrial del endometrio, es considerablemente mayor en comparación con la región antimesometrial para todos los días estudiados.

En el día 7 p.c. el epitelio luminal se prepara para la implantación embrionaria, para lo cual ocurren cambios cualitativos en la morfología de las células epiteliales de la coneja Oryctolagus cuniculus. El endometrio se observa con glándulas tubulares y rectas, presentándose más tortuosas y numerosas a partir del día 9 p.c., día en que el embrión ya está implantado, y comienza a diferenciarse. Las glándulas difieren en forma y diámetro entre ambas regiones del endometrio, experimentando un distinto crecimiento dependiendo de su ubicación mesometrial o antimesometrial, datos que se correlacionan con la forma de implantación indicada en nuestros resultados.

El trofoblasto de coneja se expande periféricamente en el día 8 de gestación, observándose la temprana aparición de los dos discos placentarios en el día 9 p.c., concordando con los resultados obtenidos por Pitt \& Carney. Según estos autores, esto ocurre más tardíamente en otras especies de roedores como el ratón. En este día, el embrión se ubicó cómodamente entre dos pliegues de la mucosa endometrial, invadiendo el lumen que quedaba entre ambos, alcanzando de esta manera regiones endometriales más profundas.

La coneja podría ser una especie usada para predecir el normal desarrollo embrionario, tras la comprensión morfológica de la implantación, por su forma poco invasiva de implantación y su capacidad de formación placentaria temprana. Proveer información de actualización en el desarrollo normal del embrión de conejo e implantación in vivo es un avance interesante para el cultivo embrionario in vitro.

Mitosis y apoptosis celular. Durante las etapas iniciales de la implantación, el epitelio uterino de ratón sufre de apoptosis en respuesta a la interacción con el blastocisto. Este proceso es esencial para que el trofoblasto logre su objetivo de entrar en una cercana asociación con el estroma endometrial durante la implantación embrionaria. La causa de la muerte celular es especulativa. Se ha sugerido que la causa podría ser una variación de $\mathrm{pH}$, autolisis o fagocitosis trofoblástica, por reacción decidual, o simplemente por muerte celular programada.

A la fecha, se han efectuado estudios de proliferación y muerte celulares, tanto de conejas no gestantes como en estado de pseudopreñez; sin embargo, existen muy pocos estudios tendientes a esclarecer la dinámica de las células del epitelio uterino, durante el periodo de pre y post implantacional en la coneja. Los estadios coordinados de muerte y proliferación celular uterina observados en coneja Oryctolagus cuniculus, podría ser sincronizada por una inducción local, guiada por la comunicación célula a célula en el período estudiado.

En coneja Oryctolagus cuniculus, el proceso de muerte celular es parte de una respuesta uterina a la preñez o es mediada por actividad trofoblástica aún no ha sido claramente establecido. Concordamos con Ferro \& Bevilacqua, al reconocer la posibilidad de la participación del trofoblasto en la modificación o incluso la aceleración del mecanismo de muerte celular.

Ohta et al.; Goodger \& Rogers y Conti et al., han descrito variaciones proliferativas en las etapas preimplantacionales, con un aumento en estas etapas y una disminución de este mecanismo durante la implantación. En la coneja Oryctolagus cuniculus, se observó una disminución en la actividad proliferativa de las células conjuntivas, estromales y las células epiteliales glandulares, entre los días 7 al 10 p.c. El pick del IA y la notoria disminución del ICM registrados, tanto a nivel del epitelio glandular como conjuntivo durante el día 8 p.c. en conejo, se relacionan directamente con 
las etapas de adhesión e invasión en este mismo día. Concordando con Galán et al. y Aplin \& Kimber, la menor actividad proliferativa observada en las células conjuntivas, tendría relación con un útero previamente receptivo, que estaría adecuándose a la implantación del blastocisto. A su vez, la mayor actividad apoptótica permitiría al trofoblasto tener acceso directo a la membrana.

Las variaciones entre los procesos de proliferación celular y apoptosis son coordinadas de modo que cuando predomina uno, otro se inhibe. Esto demuestra que la invasividad del trofoblasto en la coneja Oryctolagus cuniculus es guiada por el compartimiento endometrial, con una acción coordinada por parte de estos dos mecanismos celulares. Es así como en los procesos iniciales preimplantacionales, el epitelio uterino de la cámara de implantación de coneja se encuentra bajo un mecanismo proliferativo. Pero, a medida que avanza la implantación, el epitelio uterino sufre apoptosis en respuesta a la interacción con el blastocisto. La invasión del blastocisto y la permisividad del endometrio se deben mantener en equilibrio, para lograr el establecimiento de la placenta.

En las hembras gestantes estudiadas durante los días 7 al 10 p.c., se observó que los mecanismos de proliferación y apoptosis en los epitelios glandular y estromal se presentaban en forma paralela. Anzaldúa et al., quienes estudiaron estos procesos en el conejo, señalaron que los datos se presentaban en ambos epitelios durante la etapa preimplantacional (días 2 y 5 p.c.). Esto indicaría que tanto el epitelio glandular como estromal se comportan como una unidad funcional, que responde de manera muy similar durante los periodos pre y postimplantacional.

En coneja Oryctolagus cuniculus, las células del compartimiento estromal muestran apoptosis, detectable al mis- mo tiempo en la región antimesometrial como en la región mesometrial, y no solamente en la vecindad del trofoectodermo, como lo observado en ratón por Parr et al.; Akcali et al.; Joswig et al. y Schencke et al. en sus estudios sobre apoptosis en el epitelio uterino.

Al comparar este estudio con los realizados en ratón por Wang \& Dey (2006), es posible afirmar que en coneja Oryctolagus cuniculus, también se observa un mecanismo mesometrial-antimesometrial durante el proceso de implantación embrionaria.

En los estudios realizados por Hondo et al. y Ghaffari et. al., se señala que en el proceso de implantación de las distintas especies de mamíferos, las diferencias morfológicas y funcionales se observan a nivel del epitelio luminal, lo que se demuestra claramente en los estudios de apoptosis y mitosis realizados en coneja Oryctolagus cuniculus. Se sugiere que las glándulas uterinas guían al embrión al lugar más apropiado, el que a su vez puede estar programado genéticamente. Los estudios realizados por Lindeberg, muestran que el proceso de implantación en ferret no es comparable a rata, ratón y otros roedores.

La forma en que se implanta el conejo Oryctolagus cuniculus se relaciona con el comportamiento apoptótico observado. El epitelio uterino de la cámara de implantación de coneja Oryctolagus cuniculus se encuentra bajo un mecanismo proliferativo en los procesos iniciales de la implantación, en respuesta a la recepción del blastocisto. A medida que avanza la implantación, disminuye la proliferación y, en cambio, el epitelio uterino sufre apoptosis, en respuesta a la presencia e interacción con el blastocisto. La forma en que se implanta el conejo Oryctolagus cuniculus se relaciona con el comportamiento de proliferación y apoptosis observado en los epitelios glandular y conjuntivo endometrial.

SCHENCKE, C; ROJAS, M \& DEL SOL, M. Morphometric evaluation of the embryo-uterine relationship of the pre and post implantational stages in rabbit (Oryctolagus cuniculus). Int. J. Morphol., 26(4):995-1004, 2008.

SUMMARY: The rabbit has demonstrated to be an outstanding implanting model. As an obligated ovulating animal, its pregnancy time can be established exactly.The morphologic observation of uterus cuts of 7,8,9 and 10 days post mating, allowed this research to rebuild an apparent sequence morphometric events, that take place during rabbit implantation. Twenty New Zealand, white, adult, female rabbits were used in this research. (Oryctolagus cuniculus), nulipara. And 4, proven, fertile, males were used for mating. These males were obtained from the Bioterio of the Faculty of Medicine at the Universidad de La Frontera, Temuco, Chile. Mating day was defined as day cero, sacrificing the females days 7, 8, 9 and 10 in each mating. Once sacrificed, the pelvic region was macro and mesoscopically dissected, and the vesicles were selected for histological and immunocitochemical study Histological studies were performed with H.E. technique; morphometric and cellular kinetics with Tunnel and PCNA techniques. Descriptive statistics was used based on an average and standard deviation $(\mathrm{p}<0.001)$. The Stata 9.0 statistical program was used. The morphometric measurements obtained were: diameters and volume of the uterine vesicle, height of the uterine wall and glandular lumen of the mesometrial and antimesometrial regions to 7-10 days post coitus. The immunocitochemical analysis identified: apoptotic index of the cellular nucleous and cellular mitotic index. By day 8 post coitus were observed significant morphometric changes at the vesicular lumen, height of the uterine wall of the mesometrial region and glandular lumen. For the same day important changes of the cellular mitotic index and apoptotic index were observed.The rabbit could be a species used to predict normal embryo development, after the morphologic comprehension of the implantation, given its not so invasive form of implantation and its capacity of early placenta formation. Providing updated information in the normal development of the rabbit embryo and in vivo implantation, is an interesting move ahead for in vitro embryo cultivation.

KEY WORDS: Morphometry; Embryo-uterine relationship; Implantation; Rabbit. 


\section{REFERENCIAS BIBLIOGRÁFICAS}

Akcali, K.; Gibori, G. \& Khan, S. The involvement of apoptotic regulators during in vitro decidualization. Eur. J. Endocrinol., 149(1):69-75, 2003.

Anzaldúa, S.; Pérez, M. \& Castro, R. Variación en los índices de mitosis y apoptosis del epitelio uterino de la coneja durante los días previos a la implantación. Téc. Рecu. Méx. 39(1):59-68, 2001.

Aplin, J. \& Kimber, S. Trophoblast-uterine interactions at implantation. Reprod. Biol. Endocrinol., 5;2:48, 2004.

Conti, J.; Jiménez-Conti, I.; Conner, E.; Lehman, J. \& Gerschenson, L. Estrogen and progesterona regulation of proliferation, migration and loss in different target cells of rabbit uterine epithelium. Endocrinology, 114: 345-351, 1984.

Ferro, E. A. V \& Bevilacqua, E. Trophoblastic invasion of the uterine epithelium in Calomys callosus (Rodentia, Cricetidae). J. Morphol., 221(2):139-52, 1994.

Ghaffari, M.; Bazy, P.; Soleimani, J.; Ahmad, S.; Farzadi, L. \& Ghasemzadeh, A. Morphometric study of GnRH analog/HMG/HCG effects on ultrastructure of human endometrial epithelium in early and mid-luteal phase. $J$. Obstet. Gynaecol. Res., 33(5):681-7, 2007.

Galán, A.; O’connor, J.; Valbuena, D.; Herrer, R.; Remohí, J.; Pampfer, S.; Pellicer, A, \& Simón, C. The human blastocyst regulates endometrial epithelial apoptosis in embryonic adhesion. Biology of Reproduction, 2000.

Goodger, A. \& Rogers, P. Uterine endothelial cell proliferation before and after embryo implantation in rats. J. Reprod. Fertil., 99(2):451-7, 1993.

Hondo, E.; Phichitrasilp, T.; Kokubu, K.; Kusakabe, K.; Nakamuta, M.; Onoki, H. \& Kiso, Y. Distribution patterns of uterine glands and embryo spacing in the mouse. Anat. Histol. Embryol., 36:157-9. 2007.

Joswig, A.; Gabriel, H.; Kibschull, M., \& Winterhager, E. Apoptosis in uterine epithelium and decidua in response to implantation: evidence for two different pathways. Reprod. Biol. Endocrinol., 26:1(1):44, 2003.

Lindeberg, H. Reproduction of female ferret (Mustela putorius furo). Reprod. Dom. Anim., 43(2):150-6, 2008.
Ohta, Y.; Sato, T. \& Iguchi, T. Development and mitotic activity of the metrial gland in rat uterus during midpregnancy. In vivo, 7(2):121-6, 1993.

Parr, E; Tung, H. \& Parr, M. Apoptosis aas the mode of uterine epithelial cell death during embryo implantation in mice and rats. Biology of Reproduction, 36: 211-225, 1987.

Pitt, J. \& Carney, E. Development of a morphologicallybased scoring system for postimplantation New Zealand White rabbit embryos. Taeratology, 59(2):88-101, 1999.

Schencke, C.; del Sol, M. \& Rojas, M. Relación útero embrionaria y su variación morfológica durante el período implantacional en conejo. Int. J. Morphol. 22(4):313-22, 2004.

Wang, H. \& Dey Sk. Roadmap to embryo implantation: clues from mouse models. Nat. Rev. Genet. 7(3): 185-99. 2006.

Dirección para Correspondencia:

Prof. Mg. Cs. Carolina Schencke

Universidad Autónoma de Chile

Avda. Alemania 01090

Temuco

CHILE

Email: carolinashencke@gmail.com

Recibido : 02-07-2008

Aceptado: 12-08-2008 